\title{
Изучение многополосного экситонного спектра ZnSe в области 477-490 нм
}

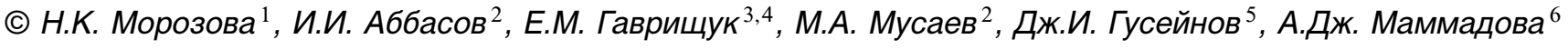 \\ ${ }^{1}$ Национальный исследовательский университет „Московский энергетический институт“, \\ 111250 Москва, Россия \\ ${ }^{2}$ Азербайджанский государственный университет нефти и промышленности, \\ AZ1010 Баку, Азербайджан \\ ${ }^{3}$ Институт химии высокочистых веществ им. Г.Г. Девятых Российской академии наук, БОКС-75, \\ 603951 Нижний Новгород, Россия \\ ${ }^{4}$ Национальный исследовательский Нижегородский государственный университет им. Н.И. Лобачевского, \\ 603022 Нижний Новгород, Россия \\ ${ }^{5}$ Азербайджанский государственный педагогический университет, \\ AZ1000 Баку, Азербайджан \\ ${ }^{6}$ Институт Биофизики, Национальной академии наук Азербайджана, \\ AZ1141 Баку, Азербайджан \\ E-mail: MorozovaNK@mail.ru, ibrahimabbasov179@gmail.com
}

Поступила в Редакцию 11 сентября 2021 г.

В окончательной редакции 16 сентября 2021 г.

Принята к публикации 16 сентября 2021 г.

Изучены спектры фотолюминесценции поликристаллического CVD (chemical vapour deposition) ZnSe, выращенного с большим избытком селена и содержащего комплексы $\left\{\mathrm{O}_{\mathrm{Se}}^{*}-\mathrm{Cu}_{i}^{+}\right\}$на дефектах упаковки. Измерено поглощение, дополняющее эти данные. Рассмотрены особенности спектров фотолюминесценции по сравнению с катодолюминесценцией. Показано, что идентичные полосы фотолюминесценции наблюдаются как несколько более коротковолновые, чем полосы катодолюминесценции. Для исследованных кристаллов представлена зонная модель согласно результатам, полученным в данной работе. Длинноволновое смещение спектров фотолюминесценции при уменьшении энергии возбуждения соответствует сдвигу по энергетической шкале зонной модели с соответствующим изменением типа излучательных переходов. Внесены изменения, определяющие природу группы эквидистантных полос 477-490 нм, характерных для образцов $\mathrm{ZnSe}$ с избытком кислорода и Se. Результаты могут быть полезны для более полного изучения структуры многофононных экситонных спектров фото- и катодолюминесценции кристаллов $\mathrm{A}^{\mathrm{II}} \mathrm{B}^{\mathrm{VI}}$.

Ключевые слова: зонная модель, узколинейчатые многофононные спектры, экситонное излучение, дефекты упаковки, изоэлектронная примесь кислорода, несущая эффективный отрицательный заряд.

DOI: 10.21883/FTP.2022.01.51816.9741

\section{1. Введение}

Селенид цинка обладает двусторонней областью гомогенности. В отличие от других халькогенидов цинка, для него характерна широкая область составов с избытком селена $\mathrm{ZnSe}(\mathrm{Se})$ [1-5]. Свечение $\mathrm{ZnSe}(\mathrm{Se})$ исследовано мало. В исследовании спектральной области краевого спектра имеются отдельные работы [4], посвященные изучению самоактивированного свечения $\mathrm{ZnSe}(\mathrm{Se})$. Для CVD (chemical vapour deposition) $\mathrm{ZnSe}$, выращенного c избытком селена в газовой фазе [2,5], обнаружены необычные узколинейчатые многофононные спектры катодолюминесценции (КЛ) в экситонной области (рис. 1). При низких температурах наблюдались эквидистантные серии полос с основными линиями серий 460, 477, 508 нм при $80 \mathrm{~K}$ [1]. По данным структурных исследований, кристаллы, несмотря на большой избыток селена, содержали кислород $\sim 10^{20} \mathrm{~cm}^{-3}$. При этом кислород за счет образования комплексов с межузельной примесью $\mathrm{Cu}_{i}^{+}$меди, присутствующей как фоновая примесь в CVD ZnSe, входил в виде устойчивых комплексов совместно с избытком селена при росте. В частности, авторы работ [6-10] на основе теории антипересекающихся зон (ВАС) рассматривали изменение зонной структуры ZnSe в зависимости от содержания кислорода.

Предпринятое нами исследование фотолюминесценции (ФЛ) таких образцов предполагало получить более полную информацию о структуре и природе таких эквидистантных полос. Преимущество фотовозбуждения состоит в глубоком проникновении возбуждающего света в объем кристалла. Это отличает спектры фотолюминесценции от КЛ и позволяет возбуждать пространственно разнесенные центры люминесценции.

Возникающая в объеме электромагнитная волна, проходя через кристалл, взаимодействует с фононами, энергия которых может переходить к экситону и обратно, а также она испытывает рассеяние на фононах [11]. Эти взаимодействия приводят к тому, что излучение (ФЛ) выходит из кристалла с измененной по сравнению с исходной длиной волны. Спектры ФЛ и их изменения в объеме в настоящее время почти не исследованы. 


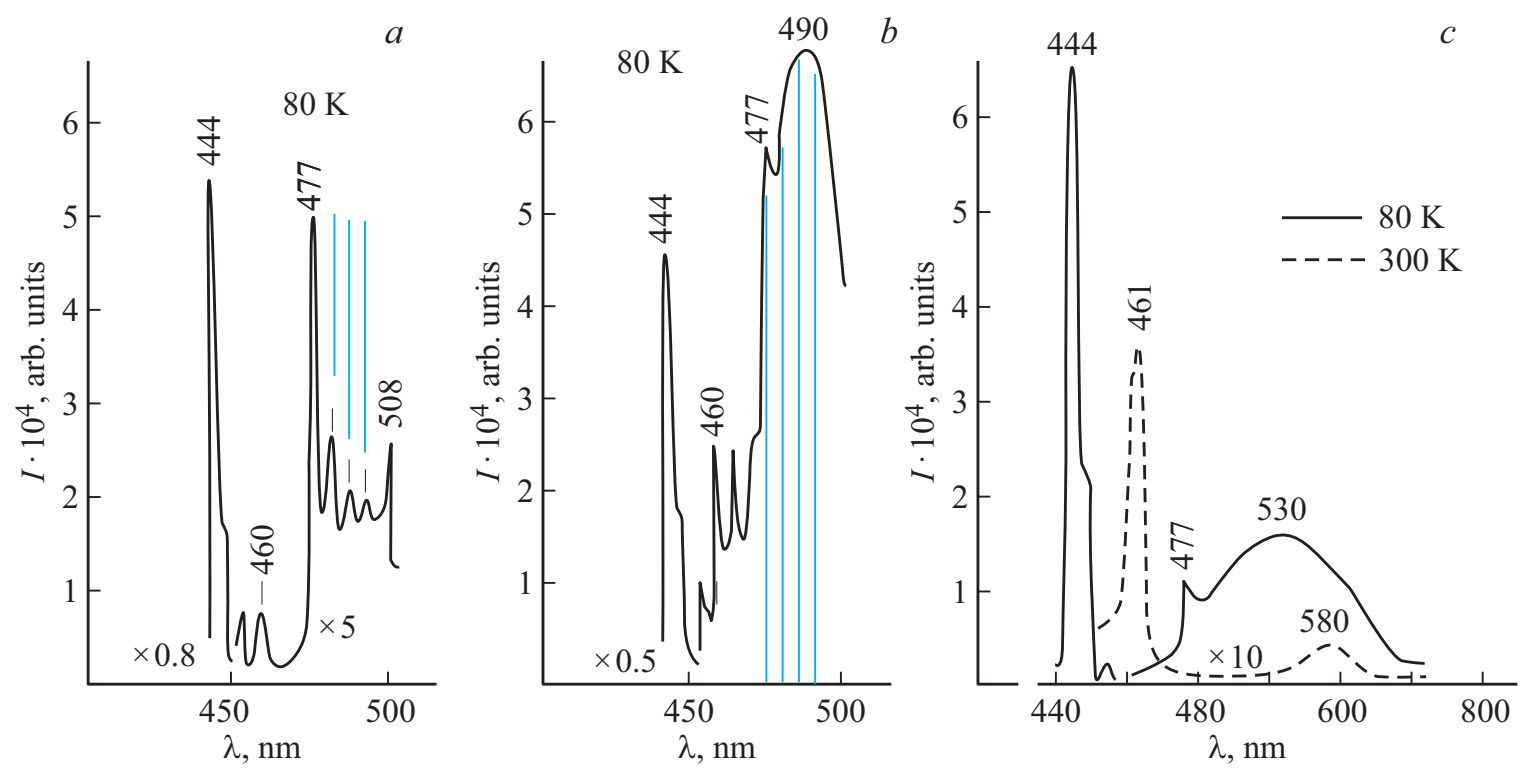

Рис. 1. Спектры КЛ разных участков $\mathrm{CVD} \mathrm{ZnSe}(\mathrm{O})$, сильно легированных в процессе осаждения кислородом и разном уровне легирования $\mathrm{Se}: a-$ с избытком $\mathrm{Se}, b-$ стехиометрического состава, $c-$ с небольшим избытком $\mathrm{Zn}[1,2]$. $\left[\mathrm{O}_{\mathrm{Se}}\right] \leq 10^{20} \mathrm{~cm}^{-3}(a)$, $\left[\mathrm{O}_{\mathrm{Se}}\right] \sim 10^{20} \mathrm{~cm}^{-3}(b, c)$.

В связи с этим нами изучена фотолюминесценция кристаллов CVD ZnSe с предварительно известным многофононным спектром прикраевой КЛ (рис. 1) [1-3].

\section{2. Методика эксперимента}

Селенид цинка был получен методом химического осаждения из газовой фазы (CVD) в Институте химии высокочистых веществ РАН России (Нижний Новгород) [12]. Для CVD $\mathrm{ZnSe}$ характерны дефекты упаковки (ДУ). Образцы при росте специально не легировались. Общее содержание примесей было $<10^{16}-10^{17} \mathrm{~cm}^{-3}$. Концентрация фоновой примеси меди $\sim 10^{16} \mathrm{~cm}^{-3}$. В обычных условиях роста растворимость кислорода в $\mathrm{ZnSe}(\mathrm{O})$ при избытке селена низкая $\leq 10^{19} \mathrm{~cm}^{-3}$ [1]. Концентрация кислорода $\left[\mathrm{O}_{\mathrm{Se}}\right]$ в исследуемых кристаллах была $\sim 10^{20} \mathrm{~cm}^{-3}$. При избытке селена и кислорода в газовой фазе в процессе роста изоэлектронная примесь кислорода $\mathrm{O}_{\mathrm{Se}}^{*}$, несущая эффективный отрицательный заряд, входит в решетку ZnSe, захватывая медь в комплексы $\left\{\mathrm{O}_{\mathrm{Se}}^{*}-\mathrm{Cu}_{i}^{+}\right\}$.

Методом количественного рентгеновского микроанализа определен состав фазы $\mathrm{ZnSe}$ и распределение химических элементов на поверхности исследуемого образца. Анализ полученных результатов показывает однородность поверхности и изменение стехиометрии в пределах области гомогенности в сторону избытка селена.

Спектры фотолюминесценции (рис. 2-4) были получены с помощью универсального флуоресцентного спектрометра (Fluo Time 300 Easy Tau). В качестве источника возбуждения использовалась ксеноновая лампа мощностью 300 Вт. Монохроматоры позволяют достичь спектрального разрешения до 0.1 нм в широком диапазоне длин волн от 200 до 1700 нм. Спектрально отфильтрованное излучение возбуждения с выходной щели монохроматора сначала коллимировалось с помощью кварцевой линзы. Данная конструкция обеспечивает высокую скорость измерения до 10 миллионов отсчетов в секунду и высокое стабильное, калиброванное по кристаллу временно́е разрешение 4 пс. Все измерения проводились при $300 \mathrm{~K}$. Спектры ФЛ регистрировались с поверхности роста на образцах размером $4 \times 4 \times 3$ мм.

УФ-видимые спектры поглощения поликристаллического CVD $\mathrm{ZnSe}$ регистрировались на установке „Specord-250“ при $300 \mathrm{~K}$ (рис. 5). Спектры (см. рис. 5) построены, в том числе с выявлением линейных участков, при использовании программы „Еxсеl“. Экстраполяция линейных участков до пересечения с осью абсцисс

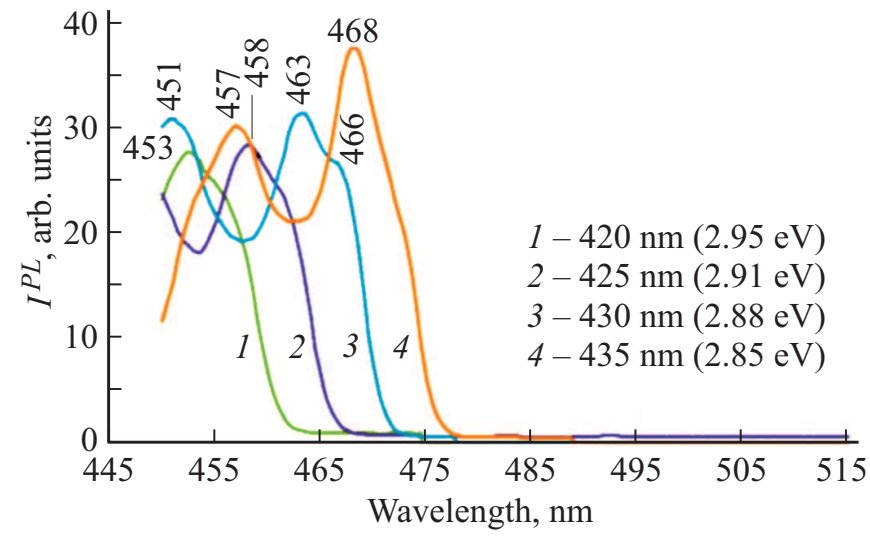

Рис. 2. Спектры фотолюминесценции поликристаллического CVD ZnSe при возбуждении разными длинами волн $420-435$ Hм. 


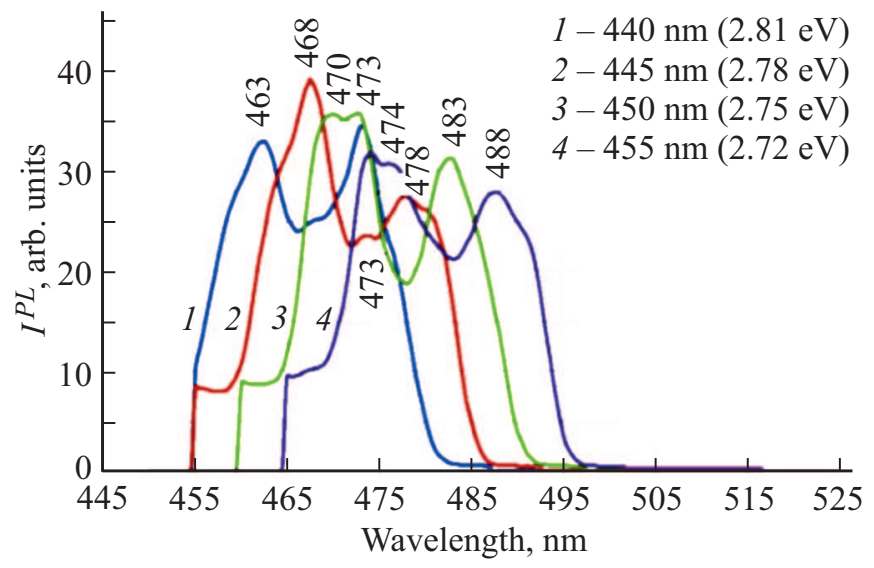

Рис. 3. Спектры фотолюминесценции поликристаллического CVD $\mathrm{ZnSe}$ при возбуждении разными длинами волн $440-455$ Hм.

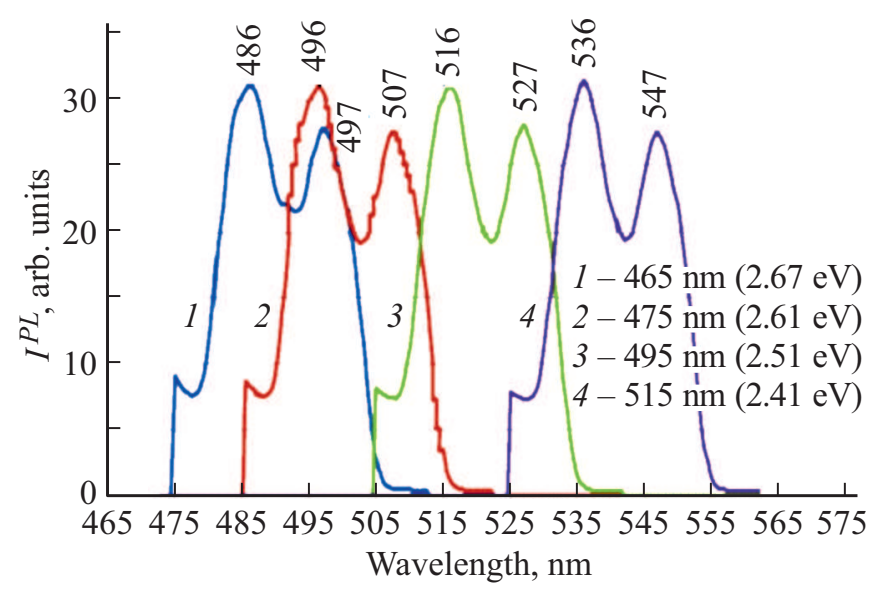

Рис. 4. Спектры ФЛ CVD ZnSe при возбуждении длинами волн: $465-515$ нм.

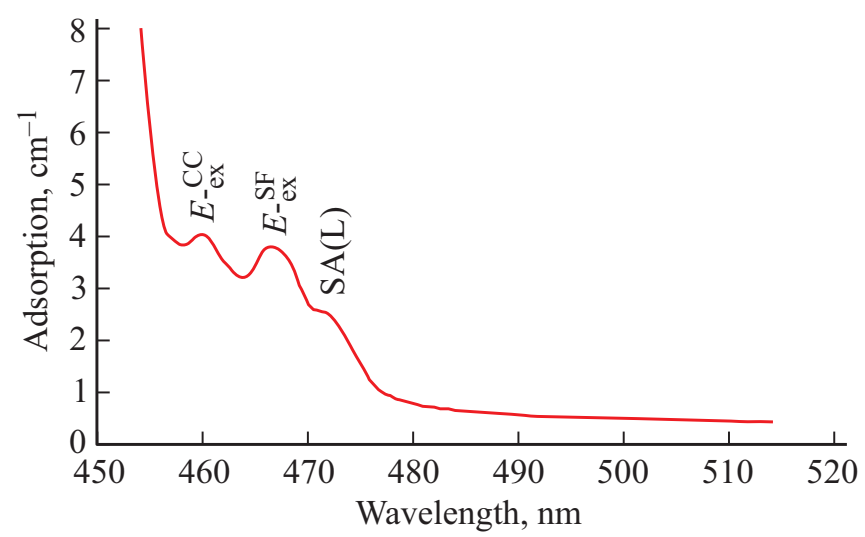

Pис. 5. Спектры поглощения поликристаллического CVD $\mathrm{ZnSe}$ при $300 \mathrm{~K}$.

также выявляет полосы поглощения. Значение ширины запрещенной зоны $\mathrm{ZnSe}$ при $300 \mathrm{~K}$ было принято 2.714 эВ $[1-3,13]$. При большой протяженности линейного участка экстраполяция дает лучшее соответствие максимуму поглощения, чем экстраполяция всего спектра поглощения.

\section{3. Экспериментальные результаты}

Исследованные нами кристаллы CVD ZnSe с избытком селена и концентрацией $\left[\mathrm{O}_{\mathrm{Se}}\right] \sim 10^{20} \mathrm{~cm}^{-3}$ стабильны за счет возникающих дефектов упаковки, сегрегирующих кислородные центры. На рис. 2-4 представлены спектры ФЛ, полученные при возбуждении мощной ксеноновой лампой на установке Fluo Time 300 Easy. Спектры сняты при изменении энергии возбуждения. На установке Time 300 можно достигнуть этого при изменении длины волны возбуждения с большой точностью.

На рис. 2 и 3 приведены данные для длин волн возбуждающего света от $420 \mathrm{Hм}$ (2.95 эВ) до $455 \mathrm{Hм}(2.72$ эВ) с интервалом, близким по энергии LO фонону ZnSe $35-38$ мэВ $[3,13]$.

При разных длинах волн накачки возникают различающиеся полосы фотолюминесценции ФЛ (рис. 2 и 3). При этом спектры ФЛ возникают начиная с возбуждения энергией $\sim 2.9$ эВ, соответствующие длинам волн 420-425 нм. При возбуждении более короткими длинами волн 400, 410, 415 нм люминесценция не наблюдалась.

Первоначально (рис. 2, кривые 1 и 2) ФЛ возникает в области свободного экситона - полоса $457 \mathrm{Hм}$ $\mathrm{ZnSe}$. C уменьшением энергии возбуждения к этой экситонной полосе присоединяются ее LO или 2LO фононные повторения (463, 468 нм и др., см. рис. 2,3), т.е. более длинноволновые фононные полосы в пределах многофононного спектра до 490 нм, соответствующие спектру КЛ (рис. 1).

С уменьшением энергии возбуждающего света от исходной 2.95 эВ до $\sim 2.8$ эВ на рис. 2 и 3 выделяется интенсивная полоса 468 нм $(\Delta \lambda \leq 20$ мэВ) (кривые 4 и 2 на рис. 2 и 3 соответственно). С изменением возбуждения намечаются фононные повторения полос: 470-473, 483, 488-486 нм (рис. 3,4), подобно многофононному спектру, который наблюдался ранее в КЛ [2], но описан как SAL(II) свечение. В кристалле возникновение этих полос связано с чистыми слоями [1].

Обратимся к модели мультизоны кристалла с дефектами упаковки (рис. 6), построенной аналогично CVD $\mathrm{ZnSe}$ в работе [3]. Учтены особенности и дополнительные данные, полученные при исследовании наших кристаллов.

Рассмотрим экспериментальные данные (спектры ФЛ) с привлечением зонной модели в соответствии с методом optical diagnostics of single-crystal systems with defects [1]. Отметки на оси ординат рис.6 соответствуют условиям съемки спектров, а точнее энергиям возбуждения ФЛ.

Из рис. $6, b$ следует, что возникновение ФЛ при 420(425) нм (см. рис. 2) связано с достижением уровня $\sim 2.9$ эВ - подзоны проводимости $E_{+}^{\mathrm{SF}}$ на дефектах 


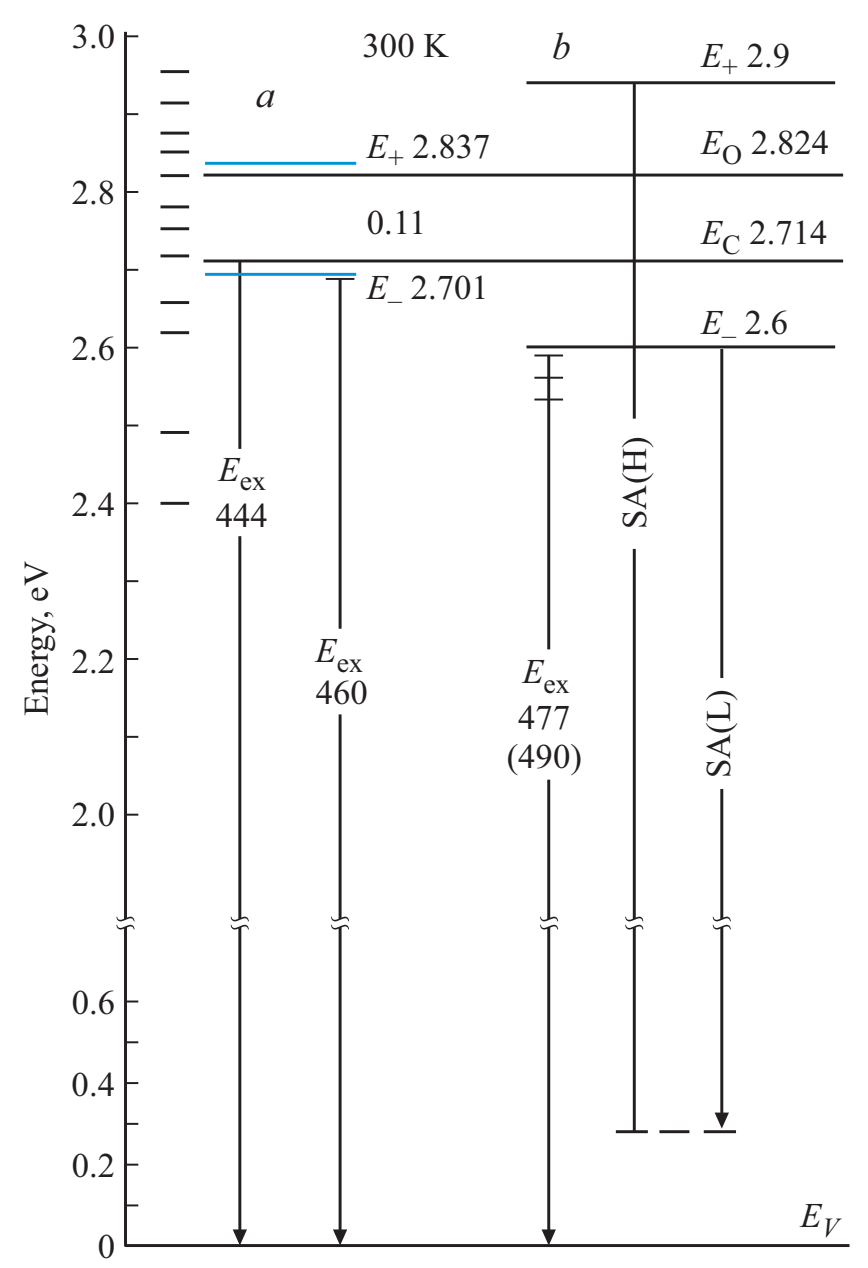

Рис. 6. Зонная модель CVD $\mathrm{ZnSe}$ с ДУ. $E_{C}=2.714$ эВ при $300 \mathrm{~K}$. Величина смещения подзон на $\sim 1$ мол\% $\left[\mathrm{O}_{\mathrm{Se}}\right]$ в $\mathrm{ZnSe}$ составляет 100 мэВ. Концентрация кислорода $\left[\mathrm{O}_{\mathrm{Se}}\right]$ в „чистых“ - светлых РЭМ слоях равна $10^{19} \mathrm{~cm}^{-3}(a)$, в слоях ДУ $-\sim 10^{20} \mathrm{~cm}^{-3}(b)$.

упаковки (stacking faults). Эта подзона возникает в соответствии с band anticrossing theory $[1,13]$ при повышенной концентрации изоэлектронных центров кислорода $\mathrm{O}_{\mathrm{Se}}^{*}$ на ДУ. Через сложную систему уровней мультизоны возможно возбуждение всего кристалла. В ФЛ при этом мы обнаруживаем экситонную полосу излучения 457 нм. Согласно схеме мультизоны (рис. 6, a) и данным [2,3] она соответствует переходам $E_{-}^{\mathrm{CC}} \rightarrow E_{V}$ от дна подзоны (2.7 эВ) проводимости чистых слоев $\mathrm{ZnSe}$.

Из-за расположения уровней $E_{-}^{\mathrm{CC}}$ в зоне, их опустошение с излучением, в частности при аннигиляции экситонов, происходит раньше других.

При возбуждении ФЛ длинами волн 435-445 нм (энергия $\sim 2.8$ эВ) (рис. 2 и 3 ) подключается второй, наиболее эффективный канал рекомбинации $E_{-}^{\mathrm{SF}} \rightarrow E_{V}-$ излучение с участием уровня дна $E_{-}^{\mathrm{SF}}$ подзоны проводимости, слоев дефектов упаковки, концентрация кислорода в котором $\sim 10^{20} \mathrm{~cm}^{-3}$. В спектрах ФЛ (рис. 2 и 3) это определяет полосу с высокой интенсивностью, 468 нм, так же как и появление ее более длинноволновых фононных повторений: 476, 488 нм (рис. 3). Эти данные при уточнении уровней зонной модели определяют экситонную полосу $E_{-}^{\mathrm{SF}} \rightarrow E_{V}$ как 477 нм при 300 К. Обращаясь к спектрам КЛ (рис. 1, b), видно, что эта полоса соответствует детально описанному в работах $[1-3,5]$ „аномальному краевому свечению“ 477-490нм. Природа аналогичных полос уточнена в спектрах $\mathrm{CdS}$ [14].

Отмеченные на рис. $6, b$ переходы $\mathrm{SA}(\mathrm{L})$ свечения соответствуют присутствию центра самоактивированного $\mathrm{SA}$ в $\mathrm{ZnSe}(\mathrm{O})$ с участием глубоких уровней рекомбинации $[1,2]$. Полоса самоактивированного свечения наблюдалась для аналогичных нашим кристаллов $\mathrm{ZnSe}(\mathrm{O}) \mathrm{Se}$ в работе [2] как полоса на длине волны 530 нм при $80 \mathrm{~K}$ (рис. $1, c)$ или как суммарная полоса $\mathrm{SA}(\mathrm{L})$ и $\mathrm{SA}(\mathrm{H})$ c максимумом $\sim 580$ нм при $300 \mathrm{~K}$. В спектрах ФЛ возникают ее составляющие - максимумы в области $440 \mathrm{Hм}$ при $300 \mathrm{~K}$ (рис. 4, кривая 4).

Если энергия возбуждения меныше чем $E_{-}^{\mathrm{SF}} \sim 2.6$ эВ, наблюдение полос излучения возможно за счет поглощения энергии SA центром. Однако благодаря составу исследуемых образцов концентрация SA центров незначительна, и при длинах волн > 515 нм спектры ФЛ мы ее не измеряли.

Представленные выводы согласуются с исследованиями спектров поглощения таких образцов. В области, достижимой для наших измерений, обнаружены три полосы (рис. 5).

Наиболее коротковолновое поглощение приходится на экситонную полосу $E_{-}^{\mathrm{CC}} 2.696$ эВ (12 мэВ) светлых слоев.

Основной максимум в спектре поглощения 2.661 эВ (рис. 5) $E_{-}^{\mathrm{SF}} \rightarrow E_{\mathrm{V}}$ соответствует экситонной полосе излучения 477 нм вблизи дна подзоны проводимости $E_{-}^{\mathrm{SF}}$. Еще более длинноволновую полосу 2.638 эВ на рис. 5 в области 470 нм можно сопоставить поглощению центром $\mathrm{SA}(\mathrm{L})$.

\section{4. Заключение}

Таким образом, измеренные полосы ФЛ непосредственно связаны с зонной моделью исследуемого кристалла. Большая глубина проникновения света по сравнению с электронным возбуждением КЛ выявляет некоторые особенности ФЛ многофононных спектров и структуру центров люминесценции в диапазоне излучения 470-490 нм. В отличие от КЛ, когда эти полосы обнаруживаются только при $80 \mathrm{~K}$, в спектрах фотолюминесценции многофононные экситонные полосы в области 477-490 нм ZnSe наблюдаются и при $300 \mathrm{~K}$.

Уточнение природы свечения 477-490 нм как ФЛ, так и КЛ $\mathrm{ZnSe}$ позволяет предполагать по аналогии с $\mathrm{CdS}$, что это излучение локализованных экситонов в решетке, предельно насыщенной кислородом.

Показано, что изменение спектра ФЛ с энергией возбуждения объясняется с позиций зонной модели 
кристалла с ДУ в соответствии с теорией антипересекающихся зон.

Длины волн полос ФЛ, присутствующих в спектрах, более коротковолновые, чем наблюдались в КЛ. Это, например, положение свободного экситона $\mathrm{ZnSe}(457$ нм $)$ по сравнению с обычными данными и спектрами КЛ (460 нм) при $300 \mathrm{~K}$.

Последнее объясняется с позиций образования поляритонов. Процессы изменения электромагнитной волны, движущейся внутри кристалла, описаны М. Кардона [11] при низких температурах. При наших измерениях это наблюдается при $300 \mathrm{~K}$. Согласно работе [11], имеет место поглощение энергии фотонного поля в объеме кристалла.

\section{Конфликт интересов}

Авторы заявляют, что у них нет конфликта интересов.

\section{Список литературы}

[1] Н.К. Морозова. Новое в оптике соединений II-VI-O: Новые возможности оптической диагностики монокристаллических систем с дефектами (Riga, Latvia, LAP LAMBERT Academic Publishing, 2021).

[2] N.K. Morozova, I.N. Miroshnikova. Semiconductors, 54 (1), $102(2020)$.

[3] В.И. Олешко, С.С. Вильчинская, Н.К. Морозова. ФТП, 55 (5), 403 (2021).

[4] R. Triboulet, J.O. Ndap, A. Tromson-Carli, P. Lemasson, C. Morhain, G. Neu. J. Cryst. Growth, 159 (1-4), 156 (1996).

[5] В.В. Блинов. Оптика центров, обязанных присутствию кислорода и меди в соединениях $\mathrm{A}^{2} \mathrm{~B}^{6}$ (на примере $\mathrm{ZnSe}$ ). Канд. дис. (М., МЭИ, 2003).

[6] W. Walukiewicz, W. Shan, K.M. Yu, J.W. Ager III, E.E. Haller, I. Miotkowski, M.J. Seong, H. Alawadhi, A.K. Ramdas. Phys. Rev. Lett., 85 (7), 1552 (2000).

[7] Jingbo Li, Su-Huai Wei. Phys. Rev. B, 73, 041201 (2006).

[8] W. Shan, W. Walukiewicz, J.W. Ager III, K.M. Yu, J. Wu, E.E. Haller, Y. Nabetani, T. Mukawa, Y. Ito, T. Matsumoto, Appl. Phys. Lett., 83 (2), 299 (2003).

[9] M.A. Mayer, Kin Man Yu, E.E. Haller, W. Walukiewicz. J. Appl. Phys., 111 (11), 113 (2012).

[10] K.M. Yu, W. Walukiewicz, J. Wu, W. Shan, J.W. Beeman, M.A. Scarpulla, O.D. Dubon, P. Becla. Phys. Rev. Lett., 91 (24), 246403 (2003).

[11] Ю.Ю. Петер, М. Кардона. Основы физики полупроводников (М., Физматлит, 2002).

[12] Г.Г. Девятых, Е.М. Гаврищук, А.Ю. Даданов. Высокочистые вещества, 2, 174 (1990).

[13] Д.А. Мидерос. Оптические свойства соединений $\mathrm{A}^{2} \mathrm{~B}^{6} c$ изоэлектронной примесью кислорода с позиций теории антипересекающихся зон. Канд. дис. (М., МЭИ, 2008).

[14] Н.К. Морозова, И.Н. Мирошникова. ФТП, 55(11), 1068 (2021).

Редактор А.Н. Смирнов

\section{Study of the multiband exciton spectrum of $\mathrm{ZnSe}$ in the range of $477-490 \mathrm{~nm}$}

N.K. Morozova ${ }^{1}$, I.I. Abbasov ${ }^{2}$, E.M. Gavrishuk ${ }^{3,4}$, M.A. Musayev ${ }^{2}$, J.I. Huseynov ${ }^{5}$, A.J. Mammadova ${ }^{6}$

${ }^{1}$ National Research University „Moscow Power Engineering Institute“, 111250 Moscow, Russia

${ }^{2}$ Azerbaijan State Oil and Industry University, AZ1010 Baku, Azerbaijan

${ }^{3}$ Institute of Chemistry of High-Purity Substances of the Russian Academy of Science, 603950 Nizhny Novgorod, Russia

${ }^{4}$ Nizhny Novgorod State University, 603950 Nizhny Novgorod, Russia

${ }^{5}$ Azerbaijan State Pedagogical University, AZ1000 Baku, Azerbaijan

${ }^{6}$ Institute of Biophysics,

National Academy of Sciences of Azerbaijan, AZ1141 Baku, Azarbaijan

Abstract The photoluminescence spectra of CVD (chemical vapour deposition) $\mathrm{ZnSe}$ polycrystalline grown with a large excess of selenium and containing $\left\{\mathrm{O}_{\mathrm{Se}}^{*}-\mathrm{Cu}_{i}^{+}\right\}$complexes at stacking faults are studied. Absorbance was measured to complement these data. The features of the PL spectra in comparison with cathodoluminescence are considered. It is shown that identical photoluminescence bands are observed as somewhat shorter wavelengths than cathodoluminescence bands. For the crystals under study, a band model is presented according to the data obtained in the given decreasing work. The low-energy shift of the photoluminescence spectra upon the excitation energy corresponds to a shift along the energy scale of the band model with a corresponding change in the type of radiative transitions. Changes have been introduced that characterize the nature of the group of equidistant bands $477-490 \mathrm{~nm}$, which are characteristic of $\mathrm{ZnSe}$ samples with an excess of oxygen and Se. The results can be useful for a more complete study of the structure of multiphonon exciton spectra of photo and cathodoluminescence of $\mathrm{A}^{\mathrm{II}} \mathrm{B}^{\mathrm{VI}}$ crystals. 